\title{
PECULIAR HORSE-SHOE KIDNEY, AND IRREGULAR LATERAL SINUSES.
}

\author{
By A. BIRMINGHAM, M.D.;
}

Professor of Anatomy, Catholic University, Ireland.

I wISH to place the following on record as irregularities of some interest :-

I. Peculiar Horse-shoe Kidney.-In a subject examined during the last winter session my attention was attracted to the unusually low position of the kidneys : the greater part of each seemed to lie in the iliac fossa, while the lower portion even reached into the true pelvis; so much was noted when the abdomen was first opened. At a subsequent stage of the dissection I found that the peculiar position of the kidneys was really due to a displaced horse-shoe kidney which lay much below the usual level. My experience of the horse-shoe variety is that the two kidneys are, as a rule, very little, if at all, displaced in a downward direction; their lower ends are simply joined by a transverse piece which crosses the front of the great vessels.

In the present case each kidney was so much displaced downwards that its middle portion lay upon the common iliac vessels, while its lower part ran down into the true pelvis, and the connecting bridge lay on the front of the sacrum.

There were, in addition, several vascular irregularities, as well as a double left ureter for a few inches from the kidney, conditions not worthy of special note.

II. Irregular Lateral Sinuses.-In this case the superior longitudinal sinus divided into nearly two equal parts at a point $1 \frac{3}{4}$ inches above the internal occipital protuberance. The straight sinus divided in a similar fashion about an inch 
from the protuberance. The corresponding branches of each trunk ran outwards towards the horizontal division of the crucial ridge, gradually approaching one another, and finally uniting, two inches from the occipital protuberance, to form the lateral sinuses. 